\title{
A novel retrieval technique for large colorectal tumors resected by endoscopic submucosal dissection: tumor extraction by defecation
}

\author{
Authors \\ Institutions \\ Daiki Nemoto' ${ }^{1}$, Yoshikazu Hayashi², Kenichi Utano', Noriyuki Isohata', Shungo Endo', Alan K Lefor ${ }^{3}$, \\ Hironori Yamamoto ${ }^{2}$, Kazutomo Togashi ${ }^{1}$ \\ ${ }^{1}$ Department of Coloproctology, Aizu Medical Center, Fukushima Medical University, Fukushima 969-3492 Japan \\ ${ }^{2}$ Department of Gastroenterology, Jichi Medical University, Tochigi 329-0498 Japan \\ ${ }^{3}$ Department of Surgery, Jichi Medical University, Tochigi 329-0498 Japan
}

Bibliography

DOI http://dx.doi.org/

10.1055/s-0041-107902

Published online: 8.1.2016

Endoscopy International Open

2016; 04: E93-E95

(c) Georg Thieme Verlag KG

Stuttgart $\cdot$ New York

E-ISSN 2196-9736

\section{Corresponding author}

Kazutomo Togashi, MD, PhD

Department of Coloproctology, Aizu Medical Center

Fukushima Medical University

Fukushima-ken, 969-3492

Japan

togashik@fmu.ac.jp
Background and study aims: Endoscopic submucosal dissection (ESD) has been developed to facilitate en bloc resection of large lesions. However, it is laborious to retrieve the large colorectal specimens. We propose a novel retrieval technique using a Valsalva maneuver, known as Tumor Extraction by Defecation (TED).

Case series: A total of nine lesions (median size 88 $\mathrm{mm}$, maximum $225 \mathrm{~mm}$; proximal colon three, rectum six) that could not be easily retrieved

\section{Introduction \\ $\nabla$}

Endoscopic submucosal dissection (ESD) has been developed to facilitate en bloc resection of large colorectal tumors $[1,2]$. Complete retrieval of the whole specimen without fragmentation is essential for ESD, because the main advantage of ESD is to allow precise evaluation of histology. With advances in ESD techniques, colorectal lesions greater than $10 \mathrm{~cm}$ have become good candidates for the procedure but it is a laborious task to retrieve such a large specimen without fragmentation. Conventional retrieval techniques (e.g. the use of net forceps [3], a small plastic bag [4] or sliding tube [5]) have been suggested, but all of them are complicated by the need for special devices, occasionally long procedure times, and possible injury to the specimen during retrieval. Consequently, a standard retrieval technique for large specimens has not yet been established. Here, we advocate a novel retrieval technique using a Valsalva maneuver, known as tumor extraction by defecation (TED). With this technique, neither special devices nor techniques are needed. TED is a simple, easy method for removing a large specimen resected by ESD. The aim of this paper is to review our experience using TED. using net forceps were subsequently removed by TED. The rectum was filled with water through the colonoscope. The patient then strained to evacuate the specimen, facilitated by an almost straight anorectal angle. All specimens were retrieved without fragmentation, within minutes. Histology was assessed appropriately, including an adenoma in two and mucosal cancer in seven. All cut margins were verified to be negative. No adverse events occurred.

\section{Case Reports \\ $\nabla$}

In two tertiary-care centers between December 2013 and June 2015, we performed ESD of 312 lesions. Fifty-four of the lesions were $50 \mathrm{~mm}$ or larger and good candidates for TED but the technique was not used for all of them. A total of nine lesions in nine patients (six men, three women; median age 70 years, range 58 to 86 ) were reviewed. ESD was carried out with carbon dioxide insufflation, under conscious sedation using intravenous pethidine hydrochloride and midazolam or dexmedetomidine hydrochloride. The resected specimen was delivered to the rectum using net forceps. Because none of the lesions were easily retrievable with net forceps, TED was attempted. After confirming that the patient was awake, the rectum was injected with $100 \mathrm{~mL}$ to $250 \mathrm{~mL}$ of water transanally through the endoscope. To evacuate the specimen, the patient strained while sitting. Rates of success with retrieval, procedure times, extent of damage to specimens, and rates of adverse events were analyzed. 


\section{Case report}

Table 1 Characteristics of nine lesions retrieved by tumor extraction by defecation (TED)

\begin{tabular}{|lllcclcl} 
No. & Location & Morphology & Tumor size $(\mathbf{m m})$ & Specimen size $(\mathbf{m m})$ & Histology & Time to extraction ${ }^{\mathbf{1}}$ \\
\hline 1 & Transverse & LST-G & $84 \times 68$ & $90 \times 72$ & Tis in adenoma & 10 \\
\hline 2 & Cecum & LST-NG & $88 \times 62$ & $90 \times 67$ & LGA & 6 \\
\hline 3 & Rectum & LST-G & $185 \times 158$ & $202 \times 168$ & Tis in adenoma & 4 \\
\hline 4 & Ascending & LST-G & $66 \times 60$ & $70 \times 65$ & Tis in adenoma & 9 \\
\hline 5 & Rectum & LST-G & $94 \times 86$ & $96 \times 88$ & Tis in adenoma & 4 \\
\hline 6 & Rectum & O-IS & LST-G & $57 \times 57$ & $60 \times 60$ & HGA & 7 \\
\hline 7 & Rectum & LST-G & $104 \times 86$ & $68 \times 62$ & Tis in adenoma & 8 \\
\hline 8 & Rectum & LST-G & $225 \times 174$ & $247 \times 188$ & Tis in adenoma & 9 \\
\hline 9 & Rectum & Tis in adenoma & 4 \\
\hline
\end{tabular}

LST-G, laterally spreading tumor, granular type; Tis, mucosal carcinoma; LST-NG: laterally spreading tumor, non-granular type; LGA, low-grade adenoma; HGA, high-grade adenoma

${ }^{1}$ Time from water injection to retrieval of the specimen, in minutes.

- Table 1 summarizes the characteristics of nine consecutive lesions retrieved using the TED technique. Seven ESDs were carried out in Aizu Medical Center Fukushima Medical University and the remaining two in Jichi Medical University Hospital. All nine specimens were completely retrieved without damage and within a short time (median 7 minutes, range 4-10 minutes) after water injection. Even lesions larger than $20 \mathrm{~cm}$ were easily extracted. The specimen histology was precisely assessed and the lesions were confirmed to be mucosal with negative margins. There were no retrieval-related complications and no post-procedure adverse events. One example is shown in $\bullet$ Fig. 1.

\section{Discussion}

$\nabla$

This is the initial report of a technique using a Valsalva maneuver to remove large colorectal specimens after ESD. All specimens were successfully retrieved within a few minutes, without complications or damage to them. Unlike conventional retrieval techniques, TED does not require special devices or specific expertise. It is simple and easy to perform in any hospital around the world. The anal canal could be a barrier to retrieving a large specimen. The anorectal angle, formed by the longitudinal axes of the anal canal and the rectum, is closely related to the defecation process. Normally, the anorectal angle ranges from 90 to 120 degrees in the resting position ( $\boldsymbol{O}$ Fig. $\mathbf{2 a}$ ). With TED, filling the rectum with water triggers the rectoanal reflex. Conscious awareness of rectal distention results in an urge to defecate. In the resting position, however, intra-abdominal pressure induced by straining decreases the anorectal angle, thus inhibiting the passage of rectal contents ( $\bullet$ Fig. $\mathbf{2 b}$ ). In the squatting position with full hip flexion (sitting position), the anorectal angle is elevated, nearly becoming straight ( $\boldsymbol{O}$ Fig. $\mathbf{2 c}$ ). Therefore, intra-abdominal pressure works effectively and increases the intrarectal pressure. Distension of the rectum also results in contraction of the rectum and simultaneous relaxation of the internal and external anal sphincters. These mechanisms allow easy passage of the large specimen through the anorectal junction ( $\bullet$ Fig. 2d) [6-8].

There are acknowledged limitations to this study. Its small size limits the generalizability of the results. Despite that, we believe that the information presented here regarding TED is useful for colonoscopists who perform ESD. TED is a promising retrieval technique for large colorectal specimens after ESD. Further study is required to confirm these observations.
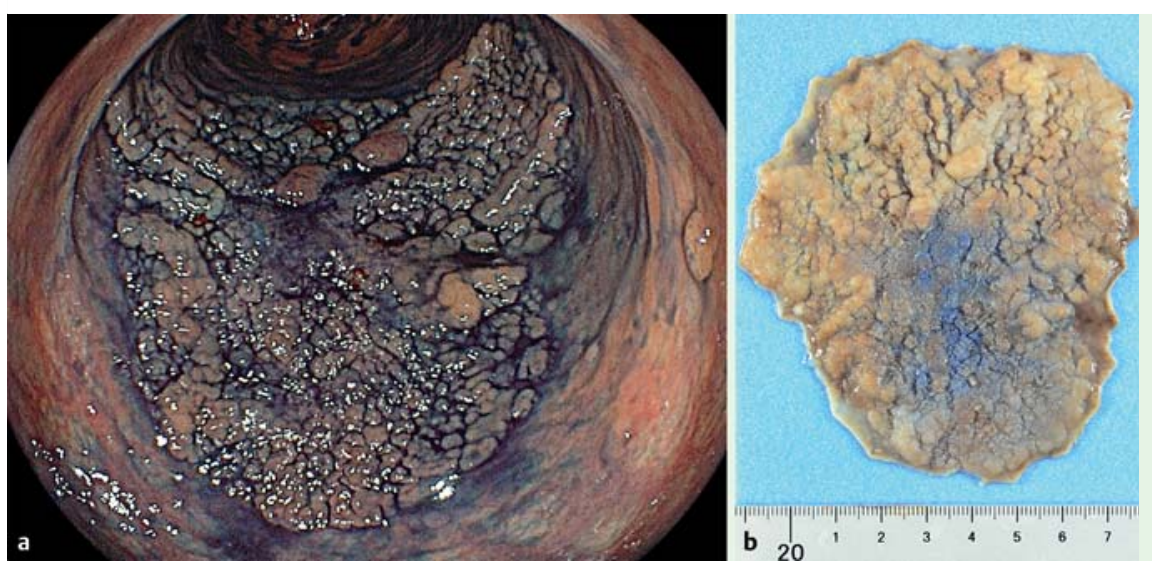

Fig. 1 Endoscopic submucosal dissection of a large laterally spreading tumor (granular-type) of the rectum. a Colonoscopy demonstrates the tumor. b The specimen is $96 \mathrm{~mm}$ long and $88 \mathrm{~mm}$ wide. Histology verified mucosal cancer in adenoma. 


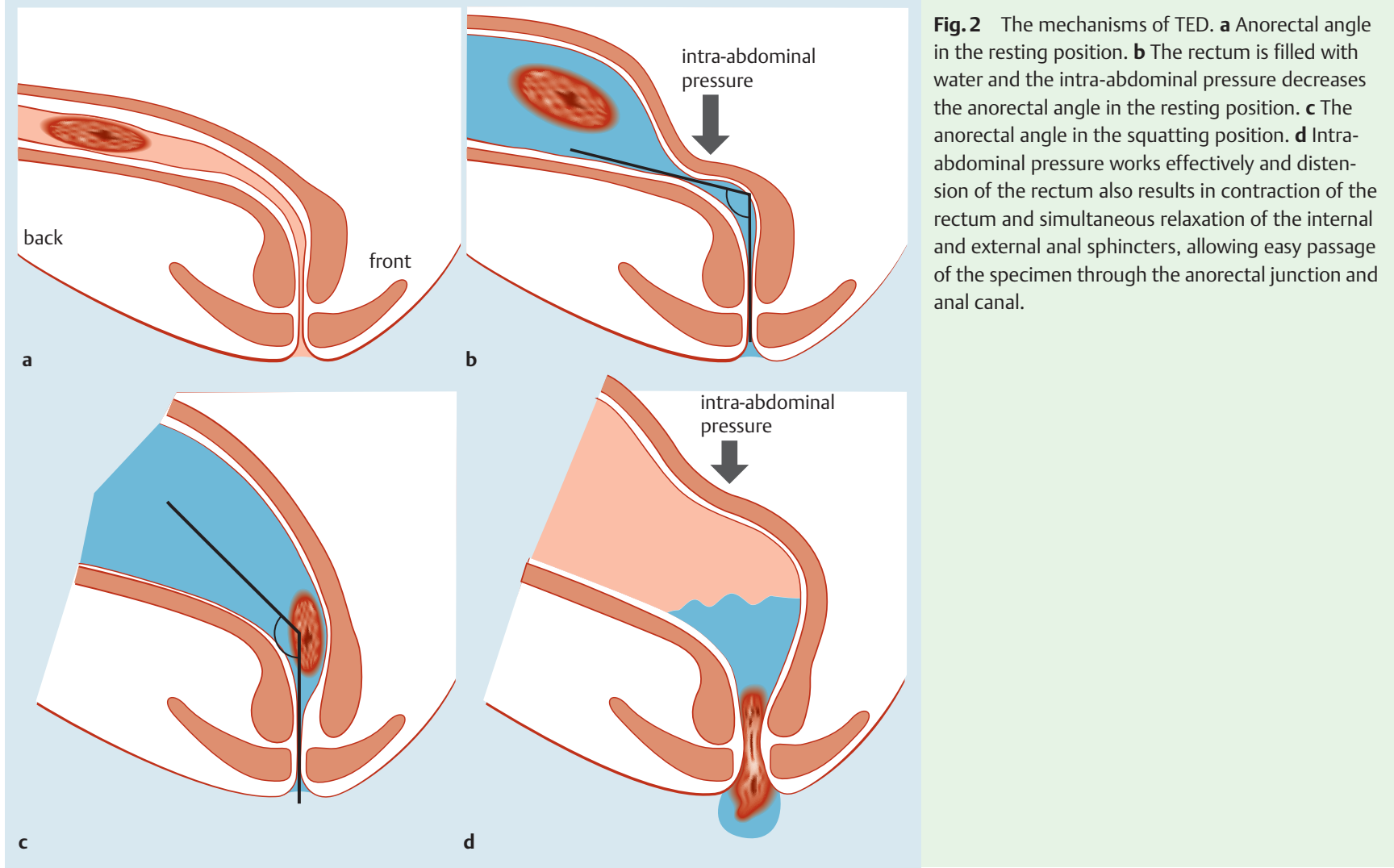

\section{Acknowledgements}

We appreciate the dedicated support provided by the nursing staff at Aizu Medical Center FMU and Jichi Medical University Hospital.

\section{References}

1 Saito Y, Uraoka T, Matsuda T et al. Endoscopic treatment of large superficial colorectal tumors: a case series of 200 endoscopic submucosal dissections (with video). Gastrointest Endosc 2007; 66: 966-973

2 Jung da H, Youn $\mathrm{YH}$, Kim JH et al. Endoscopic submucosal dissection for colorectal lateral spreading tumors larger than $10 \mathrm{~cm}$ : is it feasible? Gastrointest Endosc 2015; 81: 614-620

3 Miller K, Waye JD. Polyp retrieval after colonoscopic polypectomy: Use of the Roth Retrieval Net. Gastrointest Endosc 2001; 54: 505-507
4 Tanaka S, Toyonaga T, East J et al. Endoscopic retrieval method using a small grip-seal plastic bag for large colorectal resection specimens after endoscopic submucosal dissection. Endoscopy 2010; 42: E186187

5 Ikehara $H$, Saito $Y$, Uraoka $T$ et al. Specimen retrieval method using a sliding overtube for large colorectal neoplasm following endoscopic submucosal dissection. Endoscopy 2015; 47: E168-169

6 Rasmussen 00. Anorectal function. Dis Colon Rectum 1994; 37: 386 403

7 Irving $\mathrm{MH}$, Catchpole $\mathrm{B}$. $\mathrm{ABC}$ of colorectal diseases. Anatomy and physiology of the colon, rectum, and anus. BMJ 1992; 304: 1106-1108

8 Brookes SJ, Dinning PG, Gladman MA. Neuroanatomy and physiology of colorectal function and defaecation: from basic science to human clinical studies. Neurogastroenterol Motil 2009; 21: 9-19 